\title{
Investigation Genomic Landscapes, Molecular Mechanisms, and Extracellular Vesicles MiRNAs in Astrocyte and Oligodendrocyte Derived Amyotrophic Lateral Sclerosis
}

\author{
Ehsan Asghari Jafari \\ Islamic Azad University \\ Maryam Arabi \\ Tehran Medical Sciences Islamic Azad University \\ Ahmad Bereimipour ( $\nabla$ ahmadlta@yahoo.com ) \\ ACECR
}

\section{Research Article}

Keywords: MiRNAs, Astrocyte, Oligodendrocyte, Amyotrophic Lateral Sclerosis

Posted Date: April 12th, 2022

DOI: https://doi.org/10.21203/rs.3.rs-1404635/v2

License: (c) (i) This work is licensed under a Creative Commons Attribution 4.0 International License.

Read Full License 


\section{Abstract}

Motor neurons in the brain and spinal cord begin to die off in Amyotrophic lateral sclerosis (ALS), a disease that can be fatal. Molecular pathways in neurological disease, especially ALS, remain a challenge in the medical sciences. In this disease, a disorder in both astrocytes and oligodendrocytes can cause the disease to progress. This study aimed to investigate the molecular mechanisms and find key elements between these two cells in ALS with a bioinformatics perspective. In this study, using integrated and continuous bioinformatics analytics by various tools and databases, we investigated genes, protein products, and miRNAs between astrocytes and oligodendrocytes. The obtained data were involved in the Cellular senescence, actin cytoskeleton, cell cycle signaling pathways. Then, after careful evaluation of the information, TP53, MDM2, KRAS, PTPRC, and GSK proteins were candidates, which are regulated by hsa-miR-564, hsa-miR-496-5p, hsa-miR-324-5p, hsa-miR-296-5p, and hsa-miR-4258-3p miRNAs. Finally, the four genes had a more robust and better relationship in this study between astrocyte and oligodendrocyte derived ALS.

\section{Introduction}

An adult-onset neurodegenerative illness known as amyotrophic lateral sclerosis (ALS) is characterized by the gradual loss of motor function and, ultimately, death due to respiratory failure. ALS is the second leading cause of dementia after Alzheimer's. ALS has many neuropathological and genetic features associated with frontotemporal dementia (FTD). TDP-43, a 43-kD trans-active response (TAR) DNAbinding protein, is frequently mutated in both disorders, as are other RNA-binding proteins (RBPs) $(1,2)$.

During the past decade, the profession has been rocked by the development and clinical use of antisense oligonucleotides (ASOs). As long as the mutant gene has a toxic function, then the toxic gene product is targeted for degradation to stop the harmful RNAs or proteins in their tracks. The strategy is straightforward and elegant. As an example, in the therapy of spinal muscular atrophy, another form of oligonucleotide is employed to prevent unwanted splicing processes in order to increase gene product synthesis(2-4).

Nearly one-tenth of instances of ALS are referred to as "family-based" (FALS), while the other two-thirds are labeled "sporadic" (SALS), meaning there is no obvious family history. It has been a major factor in our understanding of disease mechanisms because of the finding of ALS-causing mutations in families and subsequent experiments on these genes. It is possible to utilize genetic methods to identify the defective gene that is associated with people who acquire ALS and separate them from people who do not. Most of the key ALS genes have been discovered as a result of this type of research(4). ALS gene discovery and validation is the first step in determining how a mutation in the protein encoded by that gene affects ALS sufferers' symptoms. Alternatively, does the mutation result in the loss of function or a gain of function (including a hazardous function). A gene therapy technique to reactivate a gene's activity would be needed if a mutation results in disease through loss of function. A therapeutic approach aimed at reducing the quantities of the mutant gene's gene products or inhibiting its functionality would be more 
appropriate if a gain in function of the mutant gene causes sickness. Sorting this data out is essential for the development of ALS therapies(5,6).

The computing and informatics difficulties in neuroscience are numerous. These issues involve a lot of "classic" bioinformatics (such as sequence analysis) that has been applied to the field of neuroscience in new and innovative ways. The word "neuroinformatics" was coined to describe the application of computers and informatics in neuroscience, probably more than any other area. There are many facets to the field of neuroinformatics, from the creation of databases to the creation of standards, models, tools, and simulations. Bioinformatics and neuroinformatics have a lot in common when it comes to studying genes and proteins $(7,8)$. A large part of the fascination with neuroinformatics, on the other hand, stems from the wide variety of neuroscience studies being conducted and the potential for these studies to be more closely linked through the use of informatics. In general, the study of molecular and genetic aspects of neurological diseases has not been done transparently and accurately so far. Examining the dimensions of signal pathways, genes, protein products produced from genes, as well as regulatory elements can provide new windows for the diagnosis and treatment of this disease $(9,10)$. Therefore, the aim of this study was to evaluate more accurately and better the microenvironmental processes in astrocytes and oligodendrocytes in patients with ALS in order to find better methods in both the diagnosis and treatment of the disease by selecting appropriate biomarkers.

\section{Results}

\section{Cellular senescence, actin cytoskeleton, cell cycle, P53 and pathways of neurodegeneration were more prominent signaling pathways astrocyte and oligodendrocyte derived ALS.}

After using the Venn tool to find the commonalities between the genes for the three groups, 404, 247, 133 high-expression genes was obtained between astrocyte and oligodendrocyte derived ALS, astrocyte derived ALS, and oligodendrocyte derived ALS respectively. These genes were involved in regulation of actin cytoskeleton, steroid hormone biosynthesis, inositol phosphate metabolism, P53 signaling pathway, cell cycle, cellular senescence, glioma, cAMP signaling pathway, protein digestion and absorption, and T cell receptor signaling pathway were observed in high-expression genes (Fig. 2A). Also, 221, 108, and 107 downregulated genes was obtained between astrocyte and oligodendrocyte derived ALS, astrocyte derived ALS, and oligodendrocyte derived ALS respectively. Regulation of lipolysis in adipocytes, insulin signaling pathway, longevity regulating pathway, aldosterone synthesis and secretion, glucagon signaling pathway, HIF-1 signaling pathway, pathways of neurodegeneration, butanoate metabolism, and tight junction observed in downregulated genes (Fig. 2B).

\section{Evaluation of gene ontology between astrocyte and oligodendrocyte derived ALS.}

This section further evaluates the astrocyte and oligodendrocyte derived ALS from the previous step and examines their molecular functions and biological processes. Accordingly, insulin-like growth factor receptor binding, hydrolase activity, hydrolyzing N-glycosyl compounds, oxidoreductase activity, acting on the $\mathrm{CH}-\mathrm{CH}$ group of donors, NAD or NADP as acceptor, retinoic acid receptor binding, FATZ binding, 
disordered domain specific binding, water transmembrane transporter activity, hydrolase activity, acting on carbon-nitrogen (but not peptide) bonds, in linear amides molecular functions in upregulated genes. Besides, pseudophosphatase activity, 15-hydroxyprostaglandin dehydrogenase (NAD+) activity, Phenanthrene 9,10-monooxygenase activity, Histone deacetylase binding, Transmembrane receptor protein tyrosine phosphatase activity, Transmembrane receptor protein phosphatase activity, and cyclohydrolase activity (Fig. 3, Table 2 and 3).

\section{Hub proteins participated in the astrocyte and oligodendrocyte derived ALS.}

In this part of the study, a more in-depth analysis of relationship between proteins with genes associated astrocyte and oligodendrocyte derived ALS was performed. KRAS, GSK, IRS1, PLCH1, AKR1C3, and IPMK in astrocyte and oligodendrocyte derived ALS were observed. TP53, MDM2, GADD45A, PKH, GAPDH, and LDHA participated in astrocyte derived ALS and PTPRC, PTK2, MLLT4, ACADS, FZD1, and ACSM2B prominent role oligodendrocyte derived ALS (Fig. 4). For more details visit the figures S1, S2, S3.

\section{Candidate critical miRNAs and extracellular vesicles' miRNAs in regulating astrocyte and oligodendrocyte derived ALS.}

In this part of the study, uploaded the genes involved in astrocyte and oligodendrocyte derived ALS to the MienTurnet database. hsa-miR-4280-3p, hsa-miR-428-5p, hsa-miR-1225-3p, hsa-miR-323b-5p, hsa-miR184-3p, hsa-miR-423-3p, hsa-miR-515-5p, hsa-miR-3559-5p, and hsa-miR-4746-3p were selected as the most important linkages with hub genes and proteins products (Fig. 5). Also showed the relationships of the other miRNAs that were significant in the miRnet database as a communication network. Then to examine the miRNAs in the extracellular vesicles, the miR-658-3p, hsa-miR-564, hsa-miR-496-5p, hsa-miR324-5p, hsa-miR-296-5p, and hsa-miR-4258-3p were more pronounced in astrocyte and oligodendrocyte derived ALS. Also hsa-miR-1244-3p, hsa-miR-409-3p, hsa-miR-496-3p, hsa-miR-658-3p, hsa-miR-423-3p, and hsa-miR-3146-3p were observed in miRnome ALS and extracellular vesicles (Fig. 6).

\section{Discussion}

The majority of the newly discovered genes have only recently been discovered and so account for a smaller percentage of the ALS population than the previously discovered genes. Optineurin is an ALSrelated gene that is directly regulated by TBK1 (TANK binding kinase 1), a protein that is necessary for autophagy and innate immune activation and is found in all cells. It has been discovered that a number of single amino acid deletions have occurred; it is probable that these are responsible for decreasing phosphorylation(11,12). After conducting a thorough examination of people suffering with ALS, researchers discovered evidence that the ALS gene TUBA4A was responsible for their condition, which was then confirmed in a second investigation. There have been cases of spinal onset with characteristic upper and lower motor neuron involvement in patients with probable mutations, albeit only a few have shown cognitive impairment or frontal lobe degeneration $(13,14)$. 
Recent investigations have discovered that loss of function mutations in the C-terminal cargo binding domain are associated with Lou Gehrig's disease (ALS). It is possible that mutation carriers cause axonal transport to be disturbed in some way, but this has not been demonstrated. ANXA11 is involved in the formation of vesicles and the anchoring of mRNA for axonal transport $(15,16)$. ALS researchers discovered a link between the gene and the disease after searching for recurrently changed genes or variants in 50 family members who had the disease. TIA mutations are known to produce a distal myopathy with vacuoles in the limbs, which has been known for a long time (just like other ALS genes VCP, MATR3, and HNRNPA2B1) $(17,18)$.

According to the findings of the research, CCNF carriers are more likely than not to also have clinical FTD in addition to their ALS. However, it was the segregating mutation (p.S621G) that exhibited the most severe effect, resulting in increased mortality of spinal cord neurons as well as a shortened length of motor axons in the laboratory $(19,20)$. No recurrent or disease-associated variations have been discovered in other cohorts, despite the presence of additional rare and novel variant types in this study's sample population. The neuropathological and clinical characteristics of mutation carriers have not yet been studied in great detail. Despite the fact that DNAJC7 haploinsufficiency is thought to be the reason for the drug's effects, no functional studies have been done $(21,22)$.

While the absence of NEK1 function or missense mutations has not yet been demonstrated, it is probable that the accumulation of DNA damage will contribute directly to cell damage and death in the absence of NEK1. LGALSL is a gene that encodes a protein that is related to galectins. However, the exact function of this protein is not known. The gene was discovered by the use of whole-exome sequencing. In comparison to the rest of the cohort, patients with LGALSL mutations experienced symptoms at an earlier age (by 13 years on average). ALS or ALS-FTD mutations have not been reported to be handed down via families $(23,24)$.

GLT8D1 has been found as a glycosyltransferase with an unknown function that has been associated with Lou Gehrig's disease (ALS) using whole-exome sequencing and candidate gene sequencing. In a study of patients who had two rare missense mutations in the ARPP21 and GLT8d1 genes, researchers uncovered a haplotype that was associated with ALS. The researchers hypothesized that the two mutations might have a synergistic influence on one another's performance. As in the instance of LGALSL, compelling evidence would indicate an etiology of ALS that had not previously been recognized $(25,26)$.

In the present study, our main focus was on the study of molecular events in astrocytes and oligodendrocytes. which also comprehensively referred to the signal pathways, the relationship between protein products and their regulators, as well as their presence in extracellular vesicles. In the following, we examined some genes and proteins. Finding these genes is likely to open new windows in the treatment of ALS.

The GSK protein produced by this gene is a serine-threonine kinase that is a member of the glycogen synthase kinase subfamily, and it is expressed in the liver. Glucose homeostasis is negatively regulated 
by this protein, which is also implicated in energy metabolism, inflammation, ER stress, mitochondrial dysfunction, and apoptotic pathways. It has been shown that mutations in this gene are linked to Parkinson's disease and Alzheimer's disease. FUS-linked ALS mutations cause cytoplasmic clumps in neurons(27). In FUS-associated ALS, they are thought to be vital. Cytoplasmic FUS aggregate clearance and breakdown in neurons may be an ALS treatment option. FUS-associated ALS is still poorly understood at the molecular level. We show that GSK-3 can modulate FUS-induced toxicity. Affecting the retina, motor neurons, and mitochondria, RNAi-mediated Shaggy knockdown in FUS-expressing flies decreases faulty phenotypes. results also showed that saggy depletion reduced cytoplasmic FUS aggregates. Co-overexpression of Slimb, an F-box protein, lowered levels of FUS proteins, demonstrating that Slimb is required for suppressing FUS-induced toxicity in Drosophila. With GSK-3 inhibitors, the SCFSlimb-mediated FUS degradation process may be changed in the real world, which could show a new way to protect the brain(28). Adults are most likely to develop ALS. Transient TDP-43

hyperphosphorylation clumps are detected in the cytoplasm of ALS patients. GSK-3 phosphorylates TDP43. The spinal cord and brain tissues of ALS patients are more active. It is possible that Tideglusib, an inhouse non-ATP competitive GSK-3 inhibitor, could be repurposed as an ALS therapeutic alternative. Thus, we evaluated Tideglusib's efficiency in multiple ALS models, both ex vivo and vitro. We detected increased GSK-3 activity, TDP-43 phosphorylation, and cytoplasmic TDP-43 accumulation in lymphoblasts from sporadic ALS patients. Inhibition of the dephosphorylation of TDP-43 by Tideglusib was reduced in ALS lymphoblasts in a human neuroblastoma model. The continuous oral Tideglusib medication lowered TDP-43 phosphorylation in the Prp-hTDP-43A315T mouse spinal cord. A phase II clinical trial for Tideglusib is planned by the end of the year. Phosphorylated GSK-3 (GSK-3) disrupts brain function, causing cell damage and disease. The GSK-3 isozymes have some overlap or compensatory activity. They are vital because GSK-3 evolved differently from other GSKs. Knockout mice and isozyme inhibitors may assist. They also demonstrated our lack of understanding of isozyme dynamics $(28,29)$. Strong GSK-3 inhibitors could treat GSK-3 hyperactivity. Which is better: isozyme inhibitors or GSK-3 inhibitors It was the same with GSK-3 knockdown (postnatal). And it may help treat FXS. It may help to stop both enzymes. To protect neurons, suppress one GSK-3 isozyme. Both GSK-3 isozymes may be more clinically efficacious. Most drugs can't pass the Blood Brain Barrier (BBB). Endothelial cells in the brain filter chemicals, peptides, and proteins. Recently, new strategies for delivering brain medications have emerged. The polymeric nanoparticles can carry drugs. It will lead to CNS therapy. Few GSK-3 inhibitors are approved. It's a new inhibitor family with a new mechanism. They may help treat neurodegenerative disorders. Most medications are unable to cross the BBB (BBB).The brain's endothelial cells act as a filter for tiny chemicals, peptides, and proteins. Various brain medication delivery methods have lately been discovered. Polymeric nanoparticles have the potential to be used as drug carriers. These new ideas will lead to CNS therapy(30-32).

In the present study, our main focus was on the study of molecular events in astrocytes and oligodendrocytes. which also comprehensively referred to the signaling pathways, the relationship between protein products and their regulators, as well as their presence in extracellular vesicles. In the following, we examined some genes and proteins. Finding these genes is likely to open new windows in 
the treatment of ALS. KRAS mutations are connected to intellectual impairment. Reduced GABAergic inhibitory neuron development and function in mutant KRAS mice has been linked to behavioral issues. The role of excitatory neurons in adult behavioral deficits is uncertain. Adult mice with constitutively active mutant KRASG12V in excitatory or inhibitory neurons have poor spatial memory. KRASG12V enhanced inhibitory GABAergic synaptic transmission. When KRASG12V is expressed in inhibitory neurons in the hippocampus, picrotoxin may restore long-term potentiation. KRASG12V destroyed excitatory neurons, possibly contributing to significant behavioral deficits. These cholinergic neurons govern learning and memory(33). Diabetes mellitus is associated with insulin resistance, or reduced neuronal metabolism and insulin signaling (AD). Insulin resistance in BFCN was expected early in AD. Insulin resistance is detected in the medial septum of 3 month old 3Tg-AD mice with AD histopathology and cognitive impairments. It was made with lots of insulin. In both circumstances, NGF decreases insulin resistance. Nutrient growth factor promotes IRS1, c-Fos, and glucose metabolism(34). Activated IRS1 on the NGF receptor TrkA is unaffected. Medial septum insulin resistance develops months before neocortex and hippocampal insulin resistance. In 3Tg-AD mice, it activates TrkA/IRS1. Treatments that target NGF and insulin receptors downstream of the insulin receptor can help reduce cognitive decline in $A D$ and diabetes-related brain insulin. Resistant insulin changes IRS-1 expression and phosphorylation. because it's unknown. Insulin resistance in peripheral organs and the brain may result from these interactions. Wnt/catenin affects IRS-1 and insulin signaling in primary neurons. Wnt3a, a Wnt agonist, boosts insulin signaling in neurons. Rat IRS-1 expression is increased in rat primary neurons by Wnt3a. Wnt3a increases insulin-mediated glucose absorption. Treatment with Wnt3a, CHIR99021, or both increases IRS-1 gene transcription and protein expression in rat primary neurons. Wnt/catenin induces IRS-1 expression. TCF4 should be linked to the 529-516 bp human IRS-1 promoter. To prevent insulin resistance, Wnt/-catenin modulates IRS-1 and insulin signaling. Neuroinflammation occurs after an ischemic stroke. Arginine is a non-essential amino acid that reduces acute inflammation(35). Arginine also reduces inflammation in ischemic brain tissue and cultured microglia following OGD injury. showed that rat I/R damage increases HIF-1 and LDHA levels, and that arginine therapy reduces HIF-1 and LDHA levels. Arginine protects against ischemic neuron death after rat I/R damage by suppressing the HIF1/LDHA-mediated inflammatory response. These findings suggest that arginine may be able to protect the brain from damage caused by cerebral ischemia by lowering the HIF-1 and LDHA-mediated inflammatory response in microglia(36). PSP has pathological and clinical Parkinson's disease symptoms. It's vital to distinguish PD from PSP. While PD and PSP biomarkers have come a long way, further biomarkers for these disorders are critically needed. 138 Parkinson's Disease Biomarkers Program participants were examined for the diagnostic potential of DUSP8 and PTPRC phosphatases. This is not the case for DUSP8 sufferers. These two biomarkers correlated, indicating they might be used together to stratify patients according to illness severity and progression. Overall, PTPRC expression may be a PD biomarker. Biomarkers like PTPRC should be researched throughout time(37).

\section{Conclusions}


Finally, genes and proteins such as GSK, IRS1, PTPRC, LDHA were more effective in protecting nerve cells. Naturally, any disruption in these genes and regulators can have irreversible consequences in weakening nerve cells. But other findings of this study could also play possible roles in the mechanism of nerve cells and the connection between synapses, which require further investigation.

\section{Materials And Methods}

\section{Select the suitable datasets and prepare the data}

In this study, bioinformatics analysis was performed. We first used the GEO database and selected the three appropriate microarray-dataset for this study. GSE26276, GSE87385 and GSE87385. After defining the groups using the GEO2R tool, we isolated the differential expression profiles of the genes and saved them in an Excel file. Then we separated the gene clusters with up and down expression and prepared them for the next step. In this part, the p value冈0.05 was considered statistically significant (figure 1 and table 1).

\section{Evaluation of signaling pathways and gene ontology:}

Genes with high and low expression in dataset were first isolated. Then uploaded the obtained common genes to the Enrichr database to examine the signaling pathways and gene ontology. After that, the KEGG library was used to analyze the signaling pathways. The ontology section was then used to evaluate the molecular functions and biological processes of high and low-expression genes. The Shiny GO database was then used to plot the communication network between the results. The $p$ value $₫ 0.05$ was considered statistically significant.

\section{Evaluation of proteins and genes network}

After evaluating the signaling pathways and gene ontology, the pathways that played a significant role in the development of neurons and ALS were selected. The relationship between their protein networks was assessed using the STRING and GeneMania databases.

\section{Select of candidate miRNAs:}

After nominating important genes and proteins in the evaluated pathways, to confirm and obtain more information about the amount of miRNAs, first uploaded the genes to the miRwalk database to validate and evaluate miRNAs. Then used the MienTurnet and miRnet databases to design the communication network between the miRNAs and the target genes.

\section{Evaluation of miRNAs released into extracellular vesicles.}

In this part of the study, using the miRnet database, we screened for miRNAs associated with extracellular vesicles. Then used exocarta and vesicle pedia databases to confirme the presence of miRNAs in extracellular vesicles more closely. 


\section{Declarations}

\section{Competing interests}

The authors declare that they have no competing interests.

\section{Authors' contribution}

E.A. M.A. A.B. Participated in study design, data collection and evaluation, drafting and statistical analysis..; Contributed extensively in interpretation of the data and the conclusion and figure design. All authors performed editing and approving the final version of this paper for submission, also participated in the finalization of the manuscript and approved the final draft.

\section{Acknowledgements}

We thank all colleagues in the core facilities for their support.

\section{References}

1. Filipi T, Hermanova Z, Tureckova J, Vanatko O, Anderova M. Glial cells-The strategic targets in amyotrophic lateral sclerosis treatment. J Clin Med. 2020;9(1):261.

2. Cruz MP. Edaravone (Radicava): a novel neuroprotective agent for the treatment of amyotrophic lateral sclerosis. Pharm Ther. 2018;43(1):25.

3. Dorst J, Ludolph AC, Huebers A. Disease-modifying and symptomatic treatment of amyotrophic lateral sclerosis. Ther Adv Neurol Disord. 2018;11:1756285617734734.

4. Andrews JA, Jackson CE, Heiman-Patterson TD, Bettica P, Brooks BR, Pioro EP. Real-world evidence of riluzole effectiveness in treating amyotrophic lateral sclerosis. Amyotroph Lateral Scler Front Degener. 2020;21(7-8):509-18.

5. Chen H, Kankel MW, Su SC, Han SWS, Ofengeim D. Exploring the genetics and non-cell autonomous mechanisms underlying ALS/FTLD. Cell Death \\& Differ. 2018;25(4):648-62.

6. Chiò A, Moglia C, Canosa A, Manera U, D'Ovidio F, Vasta R, et al. ALS phenotype is influenced by age, sex, and genetics: a population-based study. Neurology. 2020;94(8):e802--e810.

7. Klein JP, Sun Z, Staff NP. Association between ALS and retroviruses: evidence from bioinformatics analysis. BMC Bioinformatics. 2019;20(24):1-8.

8. Rana I, Rieswijk L, Steinmaus C, Zhang L. Formaldehyde and brain disorders: a meta-analysis and bioinformatics approach. Neurotox Res. 2021;39(3):924-48.

9. Mishra V, Re DB, Le Verche V, Alvarez MJ, Vasciaveo A, Jacquier A, et al. Systematic elucidation of neuron-astrocyte interaction in models of amyotrophic lateral sclerosis using multi-modal integrated bioinformatics workflow. Nat Commun. 2020;11(1):1-20.

10. Bogdahn U, Johannesen S, Huie JR, Budeus B, Peters S, Wirth AM, et al. Granulocyte-Colony Stimulating Factor: Encouraging Outcome in Sporadic ALS-Modelling and Bioinformatics Identify 
Substantial Filgrastim Responders. 2020;

11. Geser F, Brandmeir NJ, Kwong LK, Martinez-Lage M, Elman L, McCluskey L, et al. Evidence of multisystem disorder in whole-brain map of pathological TDP-43 in amyotrophic lateral sclerosis. Arch Neurol. 2008;65(5):636-41.

12. Fang T, Jozsa F, Al-Chalabi A. Nonmotor symptoms in amyotrophic lateral sclerosis: a systematic review. Int Rev Neurobiol. 2017;134:1409-41.

13. Silani V, Ludolph A, Fornai F. The emerging picture of ALS: a multisystem, not only a" motor neuron disease. Arch Ital Biol. 2017;155(4):99-109.

14. Goldstein LH, Abrahams S. Changes in cognition and behaviour in amyotrophic lateral sclerosis: nature of impairment and implications for assessment. Lancet Neurol. 2013;12(4):368-80.

15. Cirulli ET, Lasseigne BN, Petrovski S, Sapp PC, Dion PA, Leblond CS, et al. Exome sequencing in amyotrophic lateral sclerosis identifies risk genes and pathways. Science (80-). 2015;347(6229):1436-41.

16. Freischmidt A, Wieland T, Richter B, Ruf W, Schaeffer V, Müller K, et al. Haploinsufficiency of TBK1 causes familial ALS and fronto-temporal dementia. Nat Neurosci. 2015;18(5):631-6.

17. Freischmidt A, Müller K, Ludolph AC, Weishaupt JH, Andersen PM. Association of mutations in TBK1 with sporadic and familial amyotrophic lateral sclerosis and frontotemporal dementia. JAMA Neurol. 2017;74(1):110-3.

18. Le Ber I, De Septenville A, Millecamps S, Camuzat A, Caroppo P, Couratier P, et al. TBK1 mutation frequencies in French frontotemporal dementia and amyotrophic lateral sclerosis cohorts. Neurobiol Aging. 2015;36(11):3116--e5.

19. Williams KL, Topp S, Yang S, Smith B, Fifita JA, Warraich ST, et al. CCNF mutations in amyotrophic lateral sclerosis and frontotemporal dementia. Nat Commun. 2016;7(1):1-8.

20. Ohno M, Moore R, Myers P, Negishi M. Co-chaperone-mediated suppression of LPS-induced cardiac toxicity through NF\$K\$B signaling. Shock. 2018;50(2):248-54.

21. Liu X, Jian X, Boerwinkle E. dbNSFP v2. 0: a database of human non-synonymous SNVs and their functional predictions and annotations. Hum Mutat. 2013;34(9):E2393-E2402.

22. Iyer S, Acharya KR, Subramanian V. Prediction of structural consequences for disease causing variants in C21orf2 protein using computational approaches. J Biomol Struct Dyn. 2019;37(2):46580.

23. Bai SW, Herrera-Abreu MT, Rohn JL, Racine V, Tajadura V, Suryavanshi N, et al. Identification and characterization of a set of conserved and new regulators of cytoskeletal organization, cell morphology and migration. BMC Biol. 2011;9(1):1-18.

24. Forsberg K, Graffmo K, Pakkenberg B, Weber M, Nielsen M, Marklund S, et al. Misfolded SOD1 inclusions in patients with mutations in C9orf72 and other ALS/FTD-associated genes. J Neurol Neurosurg \\& Psychiatry. 2019;90(8):861-9. 
25. Filosto M, Piccinelli SC, Palmieri I, Necchini N, Valente M, Zanella I, et al. A novel mutation in the stalk domain of KIF5A causes a slowly progressive atypical motor syndrome. J Clin Med. 2019;8(1):17.

26. Gu X, Chen Y, Wei Q, Cao B, Ou R, Yuan X, et al. Mutation screening of the TIA1 gene in Chinese patients with amyotrophic lateral sclerosis/frontotemporal dementia. Neurobiol Aging. 2018;68:161e1.

27. Wirianto M, Yang J, Kim E, Gao S, Paudel KR, Choi JM, et al. The GSK-3\$ $\beta \$$-FBXL21 axis contributes to circadian TCAP degradation and skeletal muscle function. Cell Rep. 2020;32(11):108140.

28. Choi H-J, Lee JY, Cha SJ, Han YJ, Yoon JH, Kim H-J, et al. FUS-induced neurotoxicity is prevented by inhibiting GSK-3\$ $\beta$ in a Drosophila model of amyotrophic lateral sclerosis. Hum Mol Genet. 2021;

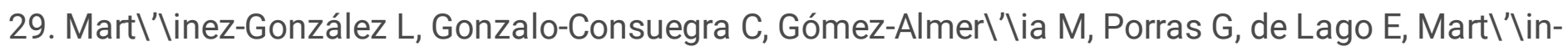
Requero Á, et al. Tideglusib, a Non-ATP Competitive Inhibitor of GSK-3\$\$\$ as a Drug Candidate for the Treatment of Amyotrophic Lateral Sclerosis. Int J Mol Sci. 2021;22(16):8975.

30. Licht-Murava A, Paz R, Vaks L, Avrahami L, Plotkin B, Eisenstein M, et al. A unique type of GSK-3 inhibitor brings new opportunities to the clinic. Sci Signal. 2016;9(454):ra110-ra110.

31. Ilouz R, Kowalsman N, Eisenstein M, Eldar-Finkelman $\mathrm{H}$. Identification of novel glycogen synthase kinase-3\$ $\beta$ S substrate-interacting residues suggests a common mechanism for substrate recognition. J Biol Chem. 2006;281(41):30621-30.

32. Balasubramaniam M, Mainali N, Bowroju SK, Atluri P, Penthala NR, Ayyadevera S, et al. Structural modeling of GSK3\$ $\beta \$$ implicates the inactive (DFG-out) conformation as the target bound by TDZD analogs. Sci Rep. 2020;10(1):1-13.

33. Ryu H-H, Kang M, Hwang K-D, Jang HB, Kim SJ, Lee Y-S. Neuron type-specific expression of a mutant KRAS impairs hippocampal-dependent learning and memory. Sci Rep. 2020;10(1):1-13.

34. Tian S, Tan S, Jia W, Zhao J, Sun X. Activation of Wnt/\$ $\beta$ \$-catenin signaling restores insulin sensitivity in insulin resistant neurons through transcriptional regulation of IRS-1. J Neurochem. 2021;157(3):467-78.

35. Sposato V, Canu N, Fico E, Fusco S, Bolasco G, Ciotti MT, et al. The medial septum is insulin resistant in the AD presymptomatic phase: rescue by nerve growth factor-driven IRS1 activation. Mol Neurobiol. 2019;56(1):535-52.

36. Chen S-F, Pan M-X, Tang J-C, Cheng J, Zhao D, Zhang Y, et al. Arginine is neuroprotective through suppressing HIF-1\$a/LDHA-mediated inflammatory response after cerebral ischemia/reperfusion injury. Mol Brain. 2020;13(1):1-13.

37. Bottero V, Santiago JA, Potashkin JA. PTPRC expression in blood is downregulated in Parkinson's and progressive supranuclear palsy disorders. J Parkinsons Dis. 2018;8(4):529-37.

\section{Tables}

Table 1: information about GEO datasets 


\begin{tabular}{|llll|}
\hline Accession ID & Name of group & Count & Platform \\
\hline GSE26276 & ALS/ASTRO/OLIGO & 9 Samples & GPL6244 \\
\hline GSE87385 & ASTROCYTE & 12 Samples & GPL570 \\
\hline GSE87385 & OLIGODENDROCYTE & 12 Samples & GPL570 \\
\hline
\end{tabular}

Table 2: molecular functions in upregulated genes between in astrocyte and oligodendrocyte derived ALS. 
Enrichment FDR

ASTRO ALS

0.0238870892703713

\section{activity}

Nucleic acid binding

Pathway

osphatase

Genes

\section{STYXL 1 STYX}

SMC1L1 EZR DDX17 ALG13 POP1 RBM28 GLRX3 PC4 PTBP3 ETF1 MACF1 WDR36 NCBP1 RPS8 C1orf131 RPS27A

\subsection{3}

0.0238870892703713

$15-$

hydroxyprostaglandin dehydrogenase (NAD+) activity

Bile acid binding AKR1C1 AKR1C3 PYGL

HPGD ABCC4
0.0368563051655137

0.0427100401626544 ALS
Phenanthrene 9,10monooxygenase activity
AKR1C1 AKR1C3

\subsection{E-07 Nucleic acid binding $\quad$ RC3H2 CCAR1 PABPC1 CFAP2O PNN ZC3HAV1 LUC7L3 CPSF6 CHD4 SPTBN1 WDR75 TIA1

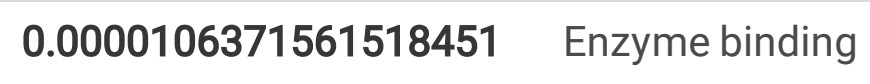 \\ 0.0000106371561518451 \\ Histone deacetylase \\ binding

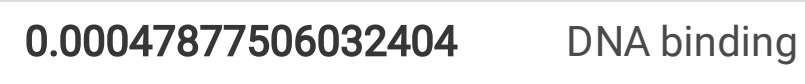 \\ 0.00047877506032404 \\ RNA binding \\ MTA3 MEF2A SUDS3 TRIM38 TRIM22 RNF217 PHF6 PPM1D TRIM27 WNK1 HACD3 RIC1 XPO5 \\ MTA3 MEF2A SUDS3 PHF6 ANKRA2 CRY1 NR2C1 KLF4 CHD4 LEF1 TP53 RBBP4 KPNA2 HDAC2 \\ CHD4 RBBP4 SMARCC1 HDAC2 ZNF195 CRY1 ZNF582 ZNF280C MEF2A ZNF506 KLF3 E2F3 ZNF684 \\ RC3H2 CCAR1 PABPC1 CFAP20 PNN ZC3HAV1 LUC7L3 CPSF6 SPTBN1 WDR75 TIA1 PRRC2C}

\section{OLIGO ALS}

\subsection{4}

Transmembrane receptor protein tyrosine phosphatase activity 


\begin{tabular}{|lll|}
$\mathbf{0 . 0 1 9 1 1 0 2 3 4 0 0 8 8 1 2 4}$ & $\begin{array}{l}\text { Transmembrane } \\
\text { receptor protein } \\
\text { phosphatase activity }\end{array}$ & PTPRO PTPRK PTPRC \\
\hline $\mathbf{0 . 0 1 9 1 1 0 2 3 4 0 0 8 8 1 2 4}$ & Cation binding & $\begin{array}{l}\text { SOD2 ESYT2 GCH1 S100A10 MTHFD2 CYLD } \\
\text { KDM4C CLEC4A METTL3 CYP2R1 RBM5 } \\
\text { variant }\end{array}$ \\
\hline $\mathbf{0 . 0 1 9 1 1 0 2 3 4 0 0 8 8 1 2 4}$ & Metal ion binding & $\begin{array}{l}\text { SOD2 ESYT2 GCH1 S100A10 MTHFD2 CYLD } \\
\text { KDM4C CLEC4A CYP2R1 RBM5 variant FAT1 } \\
\text { ZNF268 }\end{array}$ \\
\hline $\mathbf{0 . 0 2 1 9 2 6 1 6 4 1 6 7 9 0 0 1}$ & Cyclohydrolase activity & MTHFD2 GCH1 \\
\hline
\end{tabular}

Table 3: molecular functions in downregulated genes between in astrocyte and oligodendrocyte derived ALS. 
Enrichment FDR

ASTRO ALS

Pathway

Genes

insulin-like growth factor receptor binding

0.00251997219642481

0.00329972072027439

0.00417764350659283

oxidoreductase activity, acting on the $\mathrm{CH}$ -

$\mathrm{CH}$ group of donors, NAD or NADP as

acceptor

0.00567387638375119

0.00621938352769408

ubiquitin-specific protease binding

voltage-gated sodium channel activity

ALS

0.000368221934922043 nuclear receptor binding

0.000948273368847305 retinoic acid receptor binding

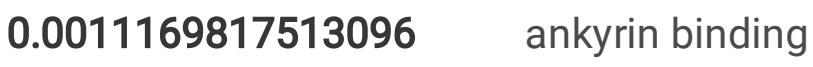

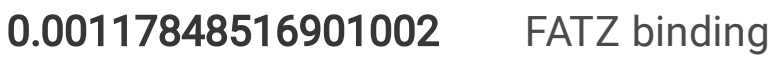

0.00197640009324868 disordered domain specific binding

OLIGO ALS

0.00191195584101257 channel activity

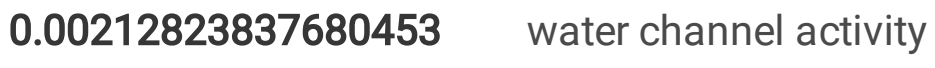

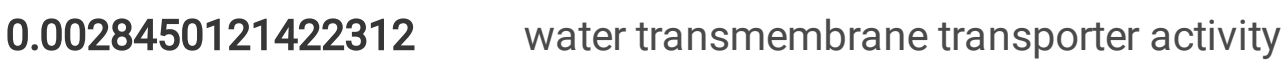

$\mathbf{0 . 0 0 3 4 9 1 9 5 8 0 6 2 3 7 8 4 \quad \text { GTP binding }}$

0.00353196021041435

hydrolase activity, acting on carbon-

nitrogen (but not peptide) bonds, in linear amides
IRS1 INSR

MACROD1 SARM1

TECR BLVRB

BAG6 RAD23A

SCN1B HCN1

ASXL 1 STAT5B NR4A1

MED25 RXRA HMGA1

MED16

ASXL 1 MED25 HMGA1

FLNC SPTB PLEC

TCAP MYOZ3

NUMA1 FKBP8 GAPDH

$A Q P 12 B A Q P 4 A Q P 1$

AQP4 AQP1

$A Q P 4 A Q P 1$

RAB1B TUBA4B TUBA4A

RHOQ DNM2

FAAH ACY1 SIRT2

\section{Figures}




\section{Figure 1}

bioinformatics analysis schematic pathway

\section{Figure 2}

In this figure, first, the shared genes between astrocyte and oligodendrocyte derived ALS via the Venn diagram was identified. Then isolated genes imvolved in crucial signaling pathways.

\section{Figure 3}

Biological processes demonstrated in upregulated and downregulated genes between A. astrocyte and oligodendrocyte derived ALS. B. astrocyte derived ALS and C. oligodendrocyte derived ALS

\section{Figure 4}

this data showed that a hub proteins involved in A. astrocyte and oligodendrocyte derived ALS. B. astrocyte derived ALS and C. oligodendrocyte derived ALS 
A

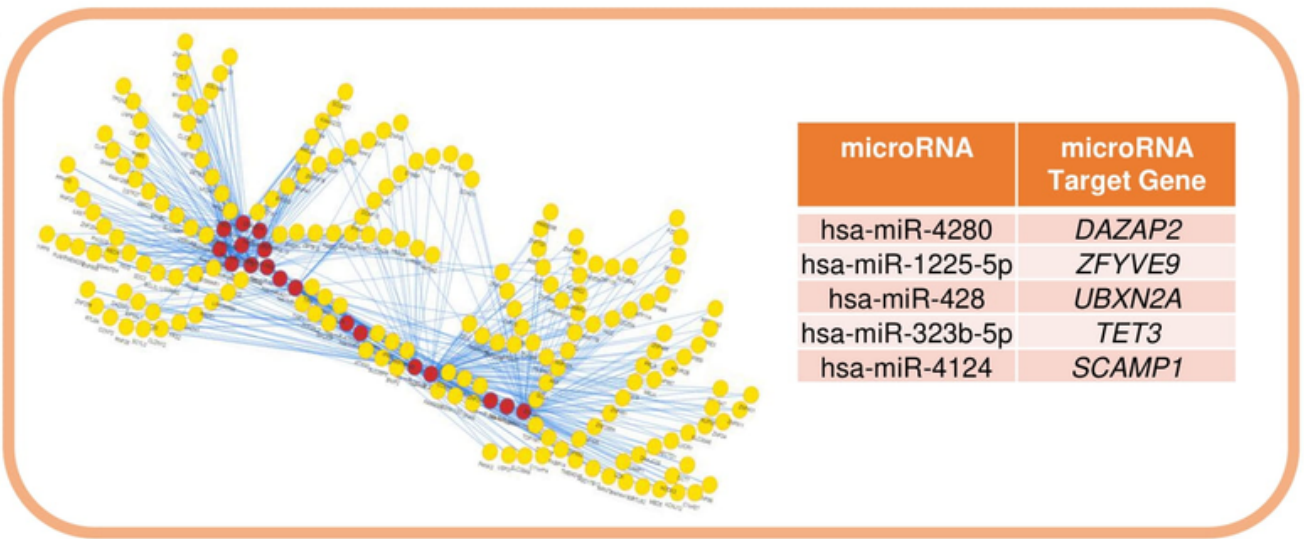

B

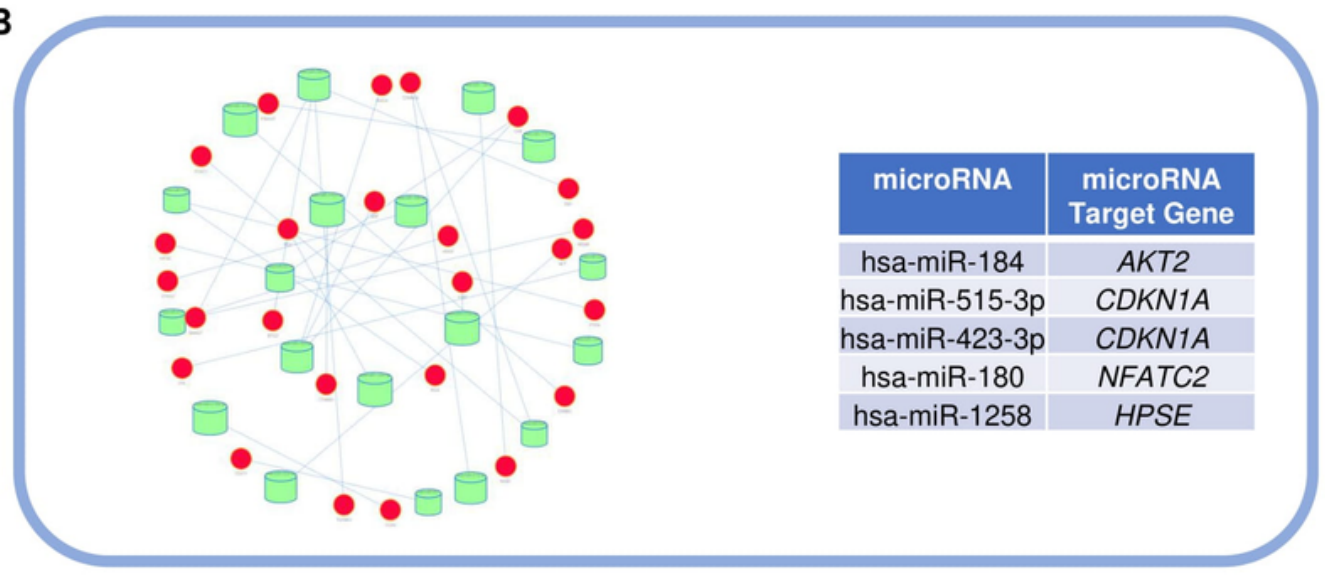

C

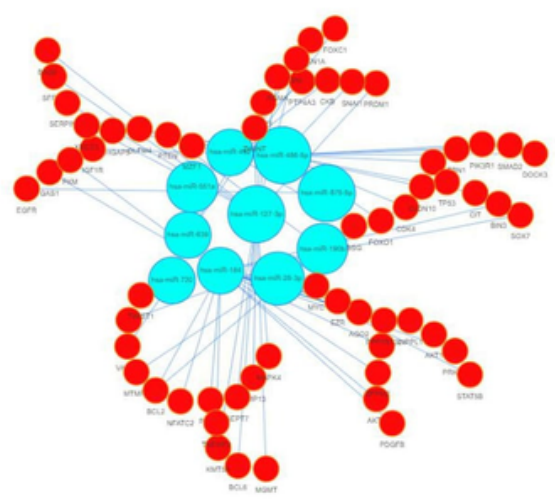

\begin{tabular}{c|c}
\hline microRNA & $\begin{array}{c}\text { microRNA } \\
\text { Target Gene }\end{array}$ \\
\hline hsa-miR-3659 & PPM1A \\
hsa-miR-591 & KATNAL1 \\
\hline hsa-miR-4746-3p & PGAM5 \\
\hline hsa-miR-4537 & PGAM5 \\
\hline hsa-miR-4098 & NLRP9 \\
\hline
\end{tabular}

\section{Figure 5}

in this part, critical miRNAs could regulated hub genes, evaluated in interactive network. A. astrocyte and oligodendrocyte derived ALS. B. astrocyte derived ALS and C. oligodendrocyte derived ALS 
Figure 6

network showed that the relationship between important extracellular vesicles miRNAs in A. astrocyte and oligodendrocyte derived ALS. B. astrocyte derived ALS and C. oligodendrocyte derived ALS.

\section{Supplementary Files}

This is a list of supplementary files associated with this preprint. Click to download.

- FIGURES1.jpg

- FIGURES2.jpg

- FIGURES3.jpg 